\title{
Neural Network Model for the Risk Prediction in Cold Chain Logistics
}

\author{
Weiyang $\mathrm{Xu}^{1}$, Zhenji Zhang ${ }^{1}$, Daqing Gong ${ }^{1 *}$ and Xiaolan Guan ${ }^{2}$ \\ ${ }^{1}$ School of Economics and Management, Beijing Jiaotong University, China \\ ${ }^{2}$ School of Economics and Management, Beijing Institute of Graphic Communication, \\ China \\ gongtuipigua@163.com \\ Abstract
}

\begin{abstract}
This study investigates environment sensitive and pertshable products (ESPPs) logistics problem, which is called cold chain logistics problem (CCLs) Based on a comprehensive literature review, we found that there is much room to improve regarding of the risks management in cold chain logistics, that is, the deyelopment of a comprehensive cold chain logistics design methodology should considered uncertainty sources and risk exposures. In this study, we propose a neural network nodel to illustrate the problems. Firstly, the paper develops input indicators at different points in cola chain logistics to examine the effects of environment fluctuations including temperature control, humidity monitoring, the temperature interruption time and electric vehicle mapping, etc; secondly, the improved neural network algorithm can achieve model convergence, including the increase of momentum term, the adjustment of ledrning rate and the change of error function. At last, through simulation, this study shows that comprehensive risk prediction of cold chain logistics will be calculated based on the input indicators using the improved neural network algorithm, and the predictive value is accurate. So not only the analyzing of kinds of cold chain logistics indicators can be realized through the Neural Network model, but we can take priorities resorting to the predictive results accordingly.
\end{abstract}

Key words: environment sensitive and perishable products (ESPPS); cold chain logistics (CCLs); input indicafors, electric vehicle; Neural Network

\section{Introduction}

For temperature sensitive and perishable products (TSPPs) logistics, a special type of supply ohain management called Cold Chain Logistics Management (CCLM), has been established. Cold chain logistics management refers to a systematic logistics project which the temperature-sensitive products are always provided in low-temperature environment in every aspect such as production, storage, transportation, marketing and consumption, to ensure quality foods, and to reduce wear and tear of logistics [1].The cold chain logistics is generally considered as the transport and storage chain between the initial production and the final consumer of temperature-controlled perishable goods, in order to ensure the quality and performance of items.

Meanwhile, cold chain logistics enterprises are important carrier. Cold chain logistics enterprises mainly include: production and processing enterprises, third-party logistics companies, sales companies and research institute. A user can map all elements of their cold

* Corresponding Author 
chain. Meanwhile, this map of the elements is a workflow diagram that outlines the associated environmental restrictions for those products, and the flow of these products from receiving, labeling, freezing, sorting, temporary storage, loading, transportation, etc.

Perishable goods, that are also temperature-sensitive products, are a fundamental source of revenue for the cold chain logistics enterprises; their management, however, constitutes a severe challenge for cold chain logistics enterprises. Typical temperature-sensitive products include dairy products, fresh food, horticultural products, blood, vaccines, pharmaceutical products and drugs, etc. [2]. A significant cost in particular is the fraction of products perished through the supply chain, which also constitutes an ethical and environmental concern [3].

It is undeniable that battery CCLM has a good future. How to manage cold chain and to know which factors can affect CCLM, as well as their impact on CCLM, that is the risk prediction in CCLM, will have a serious bearing on CCL industry development and environmental issues. Based on the findings from existing literatures, we find that existing studies mainly discuss information technology application and cold chain refrigeration technologies, etc.

The new trend of cold chain is: increased oversight, management, and control of environmental conditions across the entire supply chain; increased importance of temperature control and monitoring to mitigate and identify risks [4]. So the research questions in this study are: which factors can affect CCLM and bow they do it. At present, the aforementioned problems have not been resolved yet in related research works. In this study, we propose the neural network model to illustrate the problemis of CCLM with the help of MATLAB simulation platform. We organize this plaper as follows: in the following section, we present a comprehensive review, which forms the theoretical foundation of this study. In section 3 and Section 4, we introduce the neural network model and the improved algorithm, which provides the base for CCEM. In Section 5, we present the analytical models as well as the simulation results through which we verify the model. Finally we conclude the whole study.

\section{Literature Review}

CCL organization and operative characteristics have a significant influence on perishable goods, as in fact ensuring curtable temperature, humidity, and devices time, vehicles mapping etc. conditions for the stock-keeping units throughout the supply chain, and ensure that the ESPPs products can reach the recipients uncompromised. Cold chain constitutes four aspects of frozen processing, frozen storage, refrigerated transport and distribution, frozen sales. Storage is an mportant part of the overall cold chain, critical or major problems arising in storage are more than four times as frequent as problems arising in transport, but cold chains should always be considered as a whole if reliable results are to be obtained [5].

CCC is the outcome of the advancement of science technology and the development of refrigeration technology; especially information technology plays a critical role in the process of CCL, for example, DSS (Decision Support System) is the archetype of information technology application [6]. So Lee (2000) emphasized the importance of information resources integration[7]; Barton and Thomas(2009)pointed out rapid reaction abilities, intelligent integration system and management capabilities were all the key to create a robust supply chain[8]; Prajogo and Olhager (2011)put forward logistics resources integration concept on the background of information technology [9]. Recent developments in sensing and communication technologies allow detailed monitoring and control of CCL, all these advancements could be managed by a more efficient system for improving visibility and traceability of items in CCLM, such as, Wamba and Chatfield (2009) discussed the application of RF technology in the logistics resources integration[10]; Abad et 
al.(2009)introduced the smart tag to integrate light, temperature and humidity sensors, a microcontroller, a memory chip, low power electronics and an antenna for RFID communications[11]; Rollo and Gnoni (2010)aimed to highlight a metric model for assessing effectively economic performances of an RFID application in a specific cold chain [2]; Aung et al. (2011)presented a cold chain management system that was implemented by Radio Frequency Identification (RFID) and Wireless Sensor Network(WSN) [12]; Ting(2013)proposed a system with RFID and sensor networks to guarantee the keeping quality of low-temperature logistics [1]. So we can conclude that the quantitative model in the above literatures is information technology-based.

As a result, investments in information technology equipments applications in CCLM have helped logistics service providers to better understand the environmental variations and associated risks experienced by ESPPs, and enabled companies to address cold chain transportation challenges. However, information technology equipments are costly to cold chain logistics companies. So Montanari (2008)proposed structưred frameworks to identify the most appropriate managerial solution to be adopted in order to mininize the logistics cost[13]; a logistics service model based on the advancement of the Multi-Temperature Joint Distribution System was being proposed for the food cold chain [14]; A reliable program begun with accurate data collection of both temperature and humicity over a specific period of time [4].Not only the improvements in If are costly, but the breaks are of common occurrence in CCLM.

Breaks in the cold chain are importan economie losses in CCLM, the dangers of accidental breaks in the cold chain haye prompted studies to better analyze the risk. Matthias et al. (2007) highlighted that accídental freezing is pervasive and occurs across all segments of the vaccines cold chain[15]; many of the faitures in CCLM are due to improper adjustment of equipment parameters, such as setting the parameters for theoretical conditions, without a corresponding check in normal operation(Rodriguez, et al., 2011); for ESPPs, the principal requirement is normality maintenance of products throughout all links in cold chain, without much time breaking in CCLM, so breaks of little time may be acceptable for products, but breaks of excessive periods are likely to pose problems for products [5].So the companies that transport ESPP must be able to adjust the equipment parameters in an easy and quick way to adapt their functioning to changing environmental conditions (Rodriguez, et al.,2011).

Therefore, it is difficult lor managers to draw conclusions on the future because of high equipment cost and cold chain breaks, but users should use the existing data to determine if the cold chain system is operating within allowable tolerance levels and whether or not the cold chain process is in control. Indicators parameters play a vital role in identifying potential risks in CCLM, so the managers can take priorities when risks rise. The overall objective of CCLM is to ensure the quality and integrity of ESPPs during all aspects of the transportation [4].n one sentence, CCL should be observed at all times including storage and transportation. But an mherent risk of ESPPs is often inevitable, even in the hypothesis of perfect control with the help of information technology, so some margin for delays should be built in to the planning process; and according to Klibi et al. (2010), the development of a comprehensive SCN (Supply Chain Network) design methodology should considered uncertainty sources and risk exposures [16]. So Wirkas, et al. (2007) highlighted the low-cost and high-tech method of evaluating the cold chain function and the method should be used on a regular basis to check the integrity of the vaccine cold chain[17]; Turner et al., (2011) examined the possible impacts of policy changes on cold chain in New Zealand [18]; Joshi, et al. (2011)proposed a Delphi-AHP-TOPSIS based methodology to evaluate the cold chain performance[19]; Aiello, et al., (2012) proposed a methodology to evaluate the performance of a cold chain in terms of expected product quality based on Monte Carlo simulation [20]; Statistical analysis of the 
collected data will support the future decisions in CCLM [4]; Rediers, et al. (2009) developed indicator at different points in the supply chain to examine the effects of temperature fluctuations [21]; and a generic model to predict the remaining shelf life of meat in different steps of the supply chain was developed by Raab et al. (2008), taking into technical circumstances and temperature conditions in different supply chains [22].

In practice, multiple variables should be evaluated when making strategic decisions. Areas to be assessed include: compliance with regulations, product profile, temperature, humidity and people, etc. [4], and the indicators should include mode of transportation. In June 2006, UK funded project to "identify, develop and stimulate the development and application of more energy efficient refrigeration technologies and business practices for use throurghout the food chain whilst not compromising food safety and quality "[5]. In reality, CO2 production of cold chain in the world is likely to increase if global temperatures increase significantly [23], and with the rising concern over climate change, global warming and the recent sharp increases in energy costs, there is increasing pressure to make significant reductions in carbon emissions and energy use, especially for cold chain. Transport is responsible for a large proportion of the global emissions of climate change gases in CCLM. This has led to concerns about the long distance transport of foodstuffs. And a UK report had given estimates of $\mathrm{CO} 2$ emissions for food transport. In terms of emissions per tonne-km, the private car is the worst [5]. By the virtue of use of clean energy and reduttion of total emissions, electric vehicles become the new requirements in response to a low-carbon economy in CCLM.

By hackling these literatures, we find that there is much room for improvement regarding the associated risks when cold chain breaks; especially the production of perishable food involves a significant carbon investment in cold chain. Further study on the risk prediction in cold chain logistics is necessary tol provide intsights for practitioners and academics. In this study, we propose the neuralnetwork modet to illustrate the problems of CCLM, so the user can evaluate the potential risk between ambient and indicators for the specific situation.

\section{The Neural Network Predictive Model}

\subsection{The Standard Neural Network Model}

Neural network can study discontinuous and no-linear classification model, what is more, it has good robustness and self-adaptability; meanwhile, the proposed model should assist users to comprehend the present strengths and weaknesses of cold chain logistics, but many factors, such as the temperature excursions, humidity, storage, equipment, shipment, staff, vehicles and refrigeration technologies, etc. can play influence on cold chain logistics. So $\mathrm{BP}$ (Back Propagation)neural network is introduced,which belongs to feedforward network. $\mathrm{BP}$ neural network algorithm is formed on the basis of the gradient descent,and its learning process (training) is comprised by the forward propagation and back propagation.In forward propagation,the input information is processed layer by layer,from the input layer to the hidden layer, and transmitted to the output layer in the end.The state of the neurons in each layer affects the state of neurons the next layer only. If the outcome of the output layer is not the desired, then back propagation process begins, recursively calculating the error $E$ between the predictive value and the desired value layer by layer.By modifying the connection weights of the layers neurons, the error will get the minimum. More details in Figure 1. 


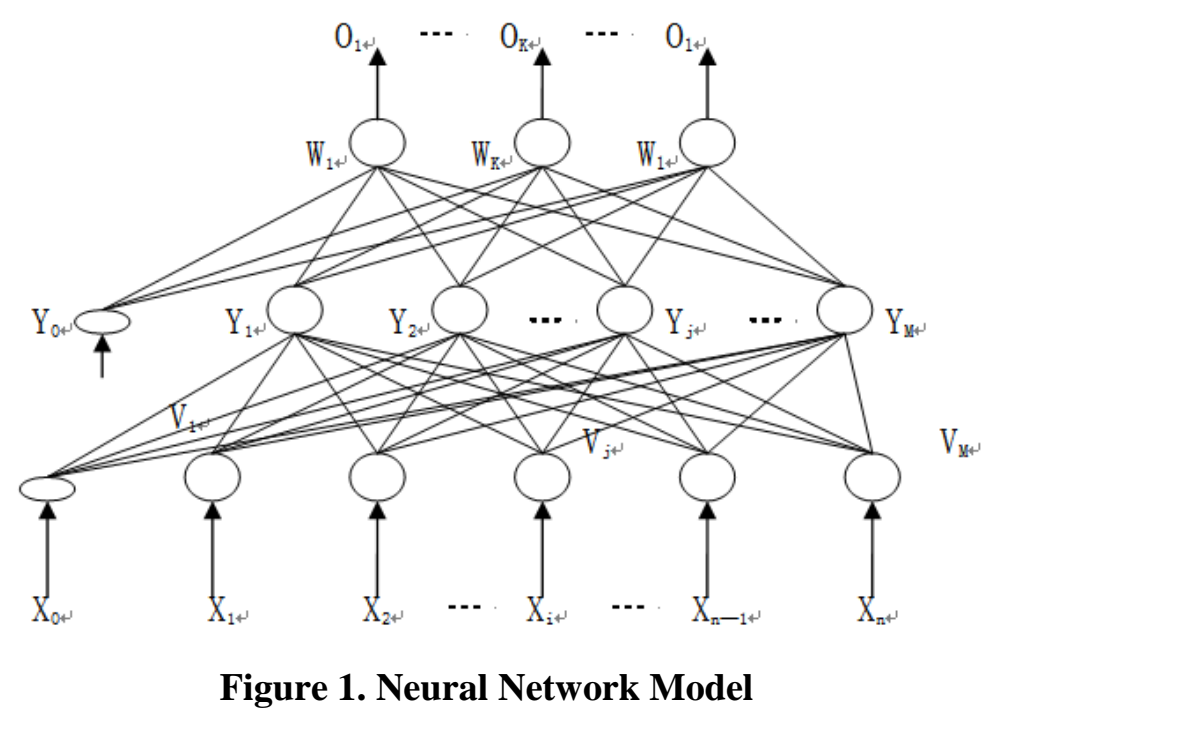

In Figure 1, the indicators vector of input layer is $X=\left(x_{1}, x_{2}, x_{i}, \cdots, x_{n}\right)^{T}$; the transition vector of the hidden layer is $Y=\left(y_{1}, y_{2}, \cdots, y, \cdots, y_{m}\right)^{\text {to }}$, the predictive value vector of the output layer is $O=\left(o_{1}, o_{2}, \cdots, o_{k}, \cdots, o_{0}\right)$ the desired value vector of the output layer is $d=\left(d_{1}, d_{2}, \cdots, d_{k}, \cdots, d_{l}\right)^{T}$.The connection weights between the input layer and the hidden layer are represented with $v$, and $V=\left(v_{q}, v\left(\ldots, v_{j}, \cdots, v_{m}\right)\right.$, where column vector $v_{j}$ is the corresponding weights vector of the $\mathrm{j}$-th reuron in the hidden layer. The connection weights between the hidden layer and the youtput layer are represented with $W$, and $W=\left(w_{1}, w_{2}, \cdots, w_{k}, \cdots, w\right)$, where column vector $w_{k}$ is the corresponding weights vector of the k-th neuron in the output layer. The mathematical model is as follows:

For the output layer:

$$
\begin{aligned}
& o_{k}=\left(\text { (net }_{k}\right) \quad k=1,2 \cdot, \cdots I, \\
& \text { Net }=\sum_{j=0}^{m} w_{j k} y_{j} \quad k=1,2 \cdot, \cdots l,
\end{aligned}
$$

For the hiadén layer

$$
\begin{aligned}
& y_{j}=f\left(\text { net }_{j}\right) \quad j=1,2 \cdot, \cdots m \\
& \text { net }_{j}=\sum_{i=0}^{n} w_{i j} x_{i} \quad j=1,2 \cdot, \cdots m
\end{aligned}
$$

Where transformation function $f(x)$ is unipolar sigmoid transformation function, and

$$
f(x)=\frac{1}{1+e^{-x}}, f^{\prime}(x)=f(x)[1-f(x)]
$$

When the predictive value vector of the output layer is not the desired, the vector error $E$ is defined as follows:

$$
E=\frac{1}{2}(d-O)^{2}=\frac{1}{2} \sum_{k=1}^{l}\left(d_{k}-o_{k}\right)^{2}
$$

Unfold the expression in hidden layer, so 


$$
E=\frac{1}{2} \sum_{k=1}^{l}\left[d_{k}-f\left(\text { net }_{k}\right)\right]^{2}=\frac{1}{2} \sum_{k=1}^{l}\left[d_{k}-f\left(\sum_{j=0}^{m} w_{j k} y_{j}\right)\right]^{2}
$$

Unfold the expression in input layer, so

$$
E=\frac{1}{2} \sum_{k=1}^{l}\left\{d_{k}-f\left[\left(\sum_{j=0}^{m} w_{j k} f\left(\text { net }_{j}\right)\right]\right\}^{2}=\frac{1}{2} \sum_{k=1}^{l}\left\{d_{k}-f\left[\left(\sum_{j=0}^{m} w_{j k} f\left(\sum_{i=0}^{n} v_{i j} x_{i}\right)\right]\right\}^{2}\right.\right.
$$

We can see that $E=F\left(w_{j k}, v_{i j}\right)$, and the change of connection weights can decrease $E$. The principle of connection weights adjustment is to decrease the $E$, so the adjustment is proportional with the gradient of $E$ :

$$
\begin{gathered}
\Delta w_{j k}=-\eta \frac{\partial E}{\partial w_{j k}} j=0 \text { 0 m } k ;=\text { D, } \cdots, l \\
\Delta v_{i j}=-\eta \frac{\partial E}{\partial v_{i j}} i=0, j ;=1, m
\end{gathered}
$$

Where the constant $\eta$ is learning rate, and $\eta \in(0,1)$. The algorithm is called Gradient Descent.

\subsection{The Neural Network Algorithm}

For input layer, Equation (9) can be changed as

$$
\Delta w_{j k}=-\eta \frac{\partial E}{\partial w_{j k}}=\eta \frac{\partial E \partial n e x_{k}}{\partial n e t \partial_{k} \partial w_{j k}}
$$

For hidden layer, Equation (10) can be changed as

$$
\begin{aligned}
& \Delta \eta_{i}=-\frac{\partial E}{\partial v_{i j}}=-p \frac{\partial E \partial n e t_{j}}{\partial n e t_{j} \partial v_{i j}}
\end{aligned}
$$

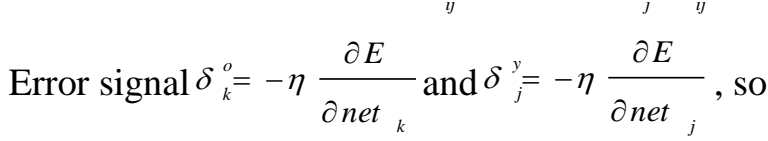

$$
\begin{aligned}
& \oslash \Delta w_{j k}=\eta \delta_{k}^{o} y_{j} \\
& \Delta w_{i j}=\eta \delta{ }_{j}^{y} x_{i} \\
& \delta_{k}=-\eta \frac{\partial E}{\partial n e t_{k}}=-\eta \frac{\partial E \partial o_{k}}{\partial o_{k} \partial n e t_{k}}=-\eta \frac{\partial E}{\partial o_{k}} f^{\prime}\left(\text { net }{ }_{k}\right) \\
& \delta_{j}^{y}=-\eta \frac{\partial E}{\partial n e t_{j}}=-\eta \frac{\partial E \partial y_{i}}{\partial y_{i} \partial n e t_{j}}=-\eta \frac{\partial E}{\partial y_{i}} f^{\prime}\left(\text { net }_{j}\right)
\end{aligned}
$$

For output layer, do differentiation for $E$

$$
\frac{\partial E}{\partial o_{k}}=-\left(d_{k}-o_{k}\right)
$$

For hidden layer, do differentiation for $E$

$$
\frac{\partial E}{\partial y_{i}}=-\sum_{k=1}^{l}\left(d_{k}-o_{k}\right) f^{\prime}\left(\text { net }_{k}\right) w_{j k}
$$

And $f^{\prime}(x)=f(x)[1-f(x)]$, so 


$$
\begin{gathered}
\delta_{k}^{o}=\left(d_{k}-o_{k}\right) o_{k}\left(1-o_{k}\right) \\
\delta_{j}^{y}=\left[\sum_{k=1}^{l}\left(d_{k}-o_{k}\right) f^{\prime}\left(\text { net }_{k}\right) w_{j k}\right] f^{\prime}\left(\text { net }{ }_{j}\right)=\left(\sum_{k=1}^{l} \delta_{k}^{o} w_{j k}\right) y_{i}\left(1-y_{i}\right)
\end{gathered}
$$

Therefore, connection weights adjustment formula are

$$
\begin{aligned}
& \Delta w_{j k}=\eta \delta{ }_{k}^{o} y_{j}=\eta\left(d_{k}-o_{k}\right) o_{k}\left(1-o_{k}\right) y_{i} \\
& \Delta v_{i j}=\eta \delta_{j}^{y} x_{i}=\eta\left(\sum_{k=1}^{l} \delta_{k}^{o} w_{j k}\right) y_{i}\left(1-y_{i}\right) x_{i}
\end{aligned}
$$

So it is easy to get that the adjustment of connection weights are decided by the learning rate $\eta$, the error signal $\delta$ and the input $Y$ or $X$. When $E$ is the desired, and we put new indicators into Neural Network model, so the risk predictive value can be got.

\section{The Improved BP Neural Network Algorithm}

The network will be unable to achieve the desired results through standard BP algorithm in many cases.The reasons are the defects of the algorithm itself: (1) Easy to form the local minimum but not the global optimum; (2) Low learning efficiency and slow convergence rate because of massive training times; (3) The falselys saturated in learning process.

Many scholars had carried out the improvements toward the defects.Some proposed modifications in the defects of algorithnitself, such a the introduction of "noise" and new error function;some used other algorithm, instead of the error gradient descent algorithm,such as conjugate gradient method and Newton method;others integrated BP algorithm with other excellent algorithm,such as genetic algorithm [24-26].This paper adopts the following measures.

\subsection{Increasing Momentum Term and the Adjustment of Learning Rate [27]}

\subsection{Transforming the Input Data and the Design of Hidden Layer}

The model cannot be convergent because of the large input data,so the input data are changed in the interval $[0,1]$. Formula is as follows:

$$
\bar{x}_{i}=\frac{x_{i}-x_{\min }}{x_{\text {max }}-x_{\text {min }}}
$$

Where $x_{i}$ is the input data, $x_{\text {min }}$ is the minimum of the input, $x_{\text {max }}$ is the maximum of the input.

Two Gidden layer cannot improve the accuracy of risk prediction compared with one hidden 1ayer (Lippman, 1987), so one hidden layer is accepted in this paper. The following formulas are used to determine the number of neurons in hidden layer:

$$
m=\sqrt{n+l}+\alpha ; m=l o g^{2 n} ; m=\sqrt{n l}
$$

Where $m$ is the number of neurons in hidden layer, $n$ is the number of neurons in input layer, $l$ is the number of neurons in output layer, $\alpha$ is the constant.

\subsection{Changing the Error Function and Transformation Function}

In the error function of standard algorithm, when $o_{k}$ is near to $1, E$ will become a constant, consequently making the network not fully trained. The reasons are as follows (takes the output layer for example): 
Error gradient $\frac{\partial E}{\partial w_{i k}}=-\delta_{k}^{o} y_{j}\left(\delta_{k}^{o}\right.$ is the error signal).Minimal error gradient means $\delta_{k}^{o}$ is closed to zero, and $\delta_{k}^{o}=\left(d_{k}-o_{k}\right) o_{k}\left(1-o_{k}\right)$.

It can be seen that $\delta_{k}^{o}$ can be closed to zero on three kinds of conditions: firstly, $o_{k}$ nearly equals $d_{k}$; secondly, $o_{k}$ is closed to 0 ; lastly, $o_{k}$ is closed to $1 . E$ can be any value if $o_{k}$ is closed to 0 or 1 , therefore the flat areas appear and the network cannot be convergent. The reason that $o_{k}$ is closed to 0 or 1 is the saturation of unipolar sigmoid transformation function $\left(f(x)=\frac{1}{1+e^{-x}}\right)$. When net input $\left|\sum_{j=0}^{m} w_{j k} y_{j}\right|$ is large enough, $o_{k}$ will be in the saturation area, and $o_{k}$ is closed to 0 or 1 .

One new error function is introduced:

$$
E_{\Sigma}=\frac{1}{2} \sum_{p=1}^{p} \sum_{k=1}^{L}\left[\left(1+d_{k}^{p}\right) \ln \frac{1+d_{k}^{p}}{1+o_{k}^{p}}+\left(1-d_{k}^{p}\right) \ln \frac{1-d_{k}^{p}}{1-o_{k}^{p}}\right] \text { (Baum, Wilozek, 1988) }
$$

One new transformation function of the neuron 18

$$
f(a)=\tanh (\quad a)=\frac{1-e^{-2 a}}{1, e^{-2 y}}=\frac{e^{a}-e^{-a}}{e^{a}+e^{-C}}
$$

Where $a$ is the input of transformation function

Because $f^{2}(a)=\tanh ^{2}(a)=\frac{e^{2}-2 e^{a} e^{-a} e^{-2 a} y}{\left(e^{a}+e^{2}\right)}=\frac{e^{2 a}-e^{-2 a}-2}{\left(e^{a}+e^{-a}\right)^{2}}$

Then

$$
\begin{aligned}
& f^{\prime}(a)=\tanh ^{\prime}(a)=\frac{\left(e^{a}+e^{-a}\right)\left(e^{a}+e^{-a}\right)-\left(e^{a}-e^{-a}\right)\left(e^{a}-e^{-a}\right)}{\left(e^{a}+e^{-a}\right)^{2}} \\
& \left(e^{\prime a}+e^{-a}\right)^{2}
\end{aligned}
$$

So $\delta_{k}^{o}=o_{k}\left(d_{k}-o\right.$, and compared with the standard error signal $\delta_{k}^{o}=\left(d_{k}-o_{k}\right) o_{k}\left(1-o_{k}\right)$, item( 1 - $\left.o_{k}\right)$ disappears. So that, the new method can avoid the "paralysis" in the training process, andaccelerate learning rate.

\section{Neural Network-based Simulation on Risk Prediction in CCLM}

\subsection{Simulation Background}

The risk prediction procedure of CCLM includes: initializing $(W$ and $V$ are endowed with random number, $E$ is $0, \alpha$ is 0.9 , training times $q$ is 1 , samples number $p$ is 1 , the interval times of training is 50, the biggest training times is 1000 , the precision of $E\left(E_{\text {min }}\right)$ is 0.05), batch training(inputting samples) and precision checking(if $E_{R M E}<E_{\min }$, the training ends; or new training begins, $\left.E_{R M E}=\sqrt{\frac{1}{P} \sum_{p=1}^{P}\left(E_{\Sigma}\right)^{2}}\right)$. More details are in Figure 2. 


\section{Figure 2. The Risk Prediction Procedure of CCLM}

The CCLM has encountered many problems through investigation (Figure1), especially in the process of temporary storage, loading and Transport. The sample enterprises are located in Beijing of China.

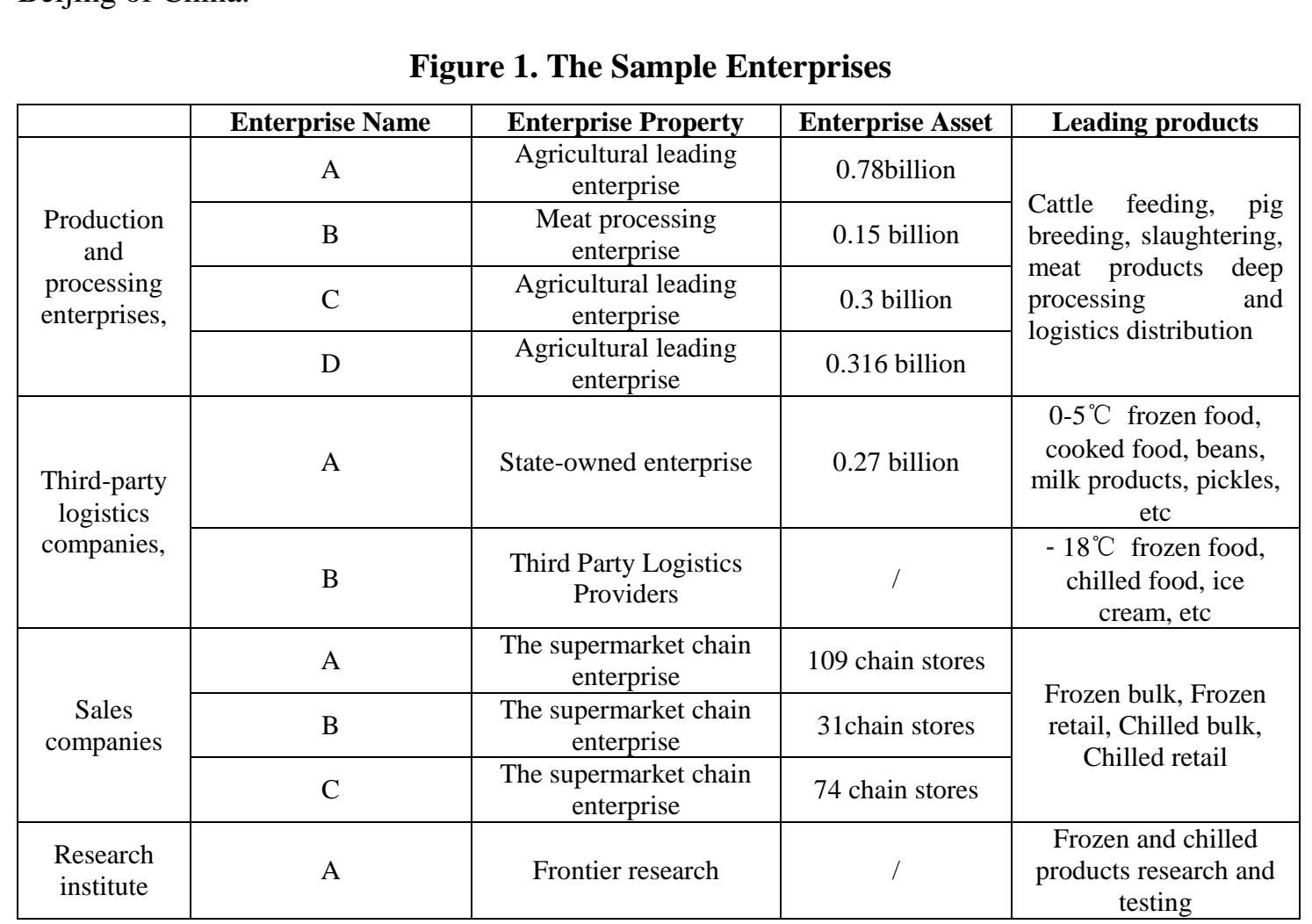


Based on the researches and investigation, the problems and needs for CCLM include: interruption of cold chain, real-time temperature monitoring is not implemented, employee theft is serious, and cargo transferring process is not standardized etc. (Gong,2013); and the existing researches focus on evaluating variation of monitoring equipment by time, temperature fluctuation, humidity, personnel training, as well as electric vehicle mapping wholly; what is more, the charging station will come about accompanying the electric vehicle, so the monitoring of electric vehicle is a must (electric vehicle's cruising radius is limited). Therefore, the need for temperature, humidity, the video interruption time, the temperature interruption time,the weighbridge measurement difference (employees weight difference between entering and leaving the warehouse), the real-time accessibility of electric vehicle tracking, etc. are all the key indicators for the cold chain logistics risk prediction.

Based on information technology, the value of indicators can be got. 80 experimental data samples are collected, 40 of which are on good condition (risk is low), 40 of which are on bad condition (risk is high).

\subsection{Network Learning and Testing}

Transformed indicators data are the input of neural network,output value is represented with 0 or 1 ( 0 means that risk is low, 1 means that risk is high), Expected indicators value range: the temperature(ralated to humidity value):-2-10 ;he humidity:-2-10\% RH;:the video interruption time: $\leq 100$ minutes; the temperature interruption time: $\leq 100$ minutes; the weighbridge measurement difference: $\leq 30$ grams; the realime accessibility of electric vehicle tracking: 0 or $1(0$ means that the vehicle is nof in control, 1 means that the vehicle is in control). 30 of the two respective samples are used to train the network,others are used to test the network.

\subsubsection{Network Training}

Coding in the Matlab(details in appendix 2):

net=newff(mimnax (P), [2,1], \{ tansig, 'tansig'\}, 'traingdx')

net.trainParam.. how $=50$; $\varnothing$

net.trainParam.epochs $=1000$;

net.trainParam.mc $=0.9$

net.trainParam.goa $=0.01$;

$1 \mathrm{r}=0.01$;

$1 r \_$inc $=1.05 \cdot 1$

$1 \mathrm{r} \_$dec $=0.7$,

err $\mathrm{rat10}=1.04$;

[net, tr] =train $($ net, $\mathrm{P}, \mathrm{T})$;

$\mathrm{A}=\operatorname{sim}($ net, $\mathrm{P})$

So the error curve is got by the code above (Figure 3 ). 


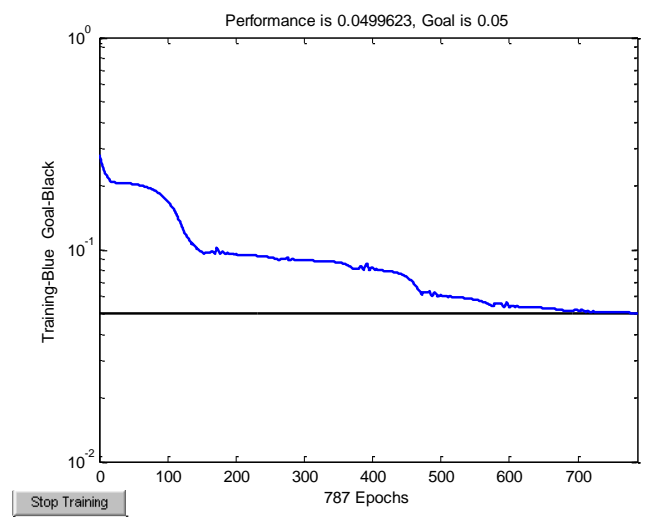

Figure 3. Error Curve

In Figure 3,the precision of error is meet after 787 times

\subsubsection{Network Testing}

$\mathrm{P} 1$;

$\mathrm{A}=\operatorname{sim}($ net,P1)

Load the trained network, and input the testing samples, then the predictive results of risk are $\operatorname{got}($ Figure 4$)$.

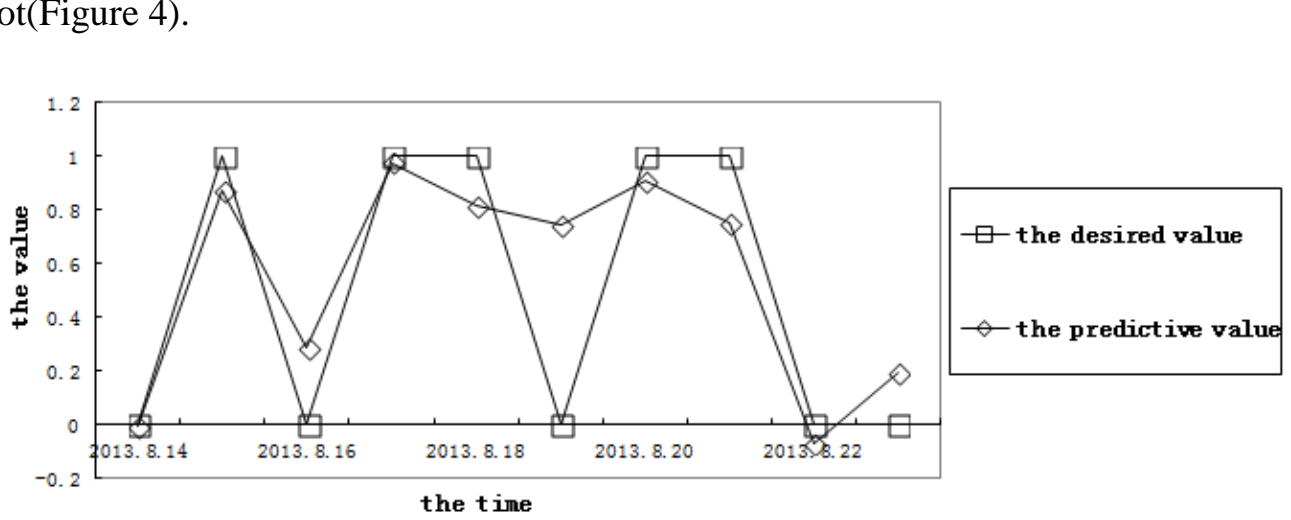

Figure 4. The Predictive Results

Figure 4 graphically depicts the performance of ten samples based on the indicators data over a particular time frame. When considering this simulaiton results, the line-graph clearly outlines the predictive results are basically accurate, except for the "2012.8.19". There are closed quantitative relationships between samples and indicators.More indicators needs more corresponding samples. In the case of less samples but more indicators, the predictive results are still satisfactory. So the topological graph of risk prediction is got based on the indicators and neural network algorithm, more details are seen in Figure 5. 


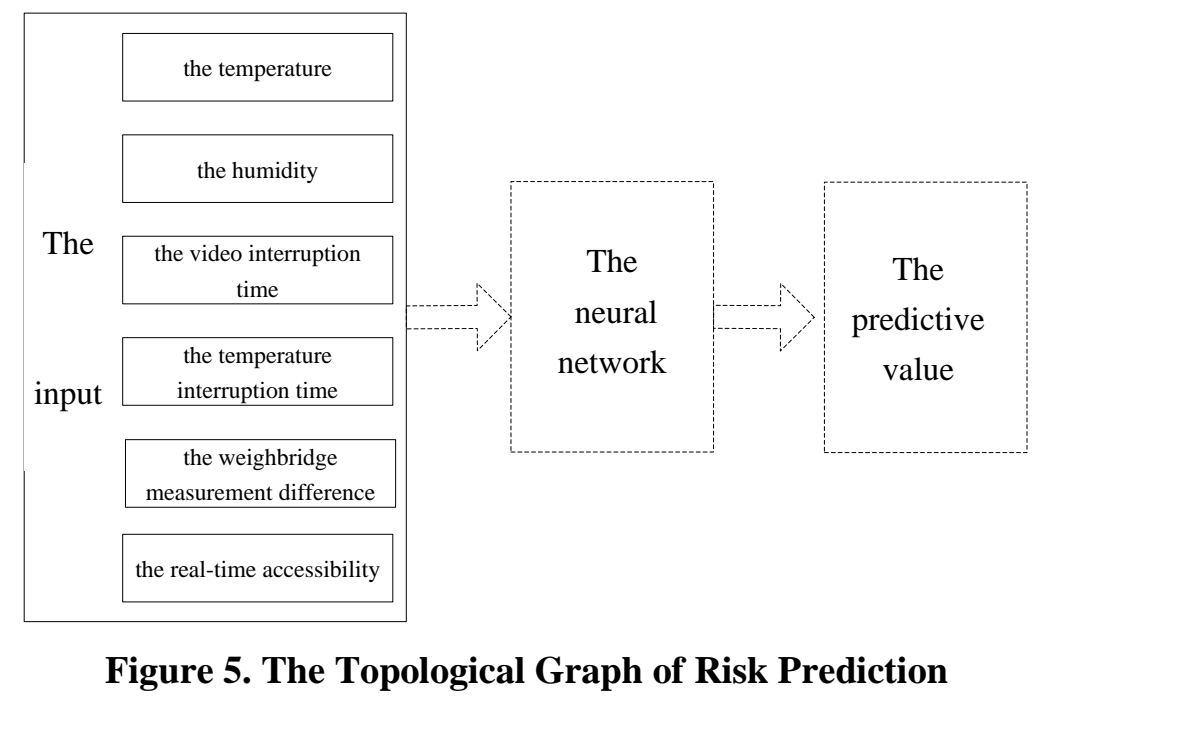

In Figure 5, we can get the new predictive value by the normalization of the new input(the temperature,the humidity,the video interruption cime,the temperature interruption time, the weighbridge measurement difference, the real-time accessibility of electric vehicle tracking) in CCLM.

Therefore, cold chain documentation of gisk prediction can help to prove that a system is operating in a controlled manner; it capalso provide evidence to show how a suspect environment fluctuation is evaluated and what final decisions are made regarding the risk correspondingly. So it is critical that the neural network application of risk prediction provide the capability to create and share reports both internally and with supply chain partners in CCLM.

\section{Conclusions}

The cold chain is generally considered as the transport and storage chain between the initial production and the final consumer of temperature-controlled perishable goods (Heap, 2006). This consists of a series of storage and transport links, all of which are designed to keep the products on the correct condition untily them reach the recipient. So many researches are developed to improve visibility and traceability of CCLM based on information technology (Radio Frequency Identification and Internet of Things) applications, thus supporting the design and the control of the whole cold supply chain. However, because of the cost, breaks of cold chain and environment protection, it is dtill difficult for users to draw conclusions on the future based only on the existing data, or the users cannot take priorities when risks rise.

The study focuses on the risk prediction of CCLM. On one hand, some items are developped to indicaitos, ncluding temperature, humidity, the video interruption time, the temperature interruption time, the weighbridge measurement difference, the real-time accessibility of electric vehicle tracking, etc., so that we can achieve the increased oversight, management, and control of environmental conditions across the entire supply chain; On the other hand, the improved neural network algorithm is introduced, such as increasing momentum term and the adjustment of learning rate, the input data transformation and the design of hidden layer, changing the error function and transformation function, etc.,not only can the improved algorithm overcome the slow convergence problem, but monitor and identify risks in CCLM.

Of course, like all of the researches, this paper has certain limitations and deficiencies. It requires a lot of raw data for simulation, but the simulation studies are carried out based on laboratory data instead of raw data, and it does not compare the method with other methods. 


\section{Acknowledgement}

The paper is supported by National Natural Science Foundation(71132008), the Fundamental Research Funds for the Central Universities(B12JB00280) and Beijing Municipal Science \& Technology Commission(Z121100000312093). I will appreciate them.

\section{References}

[1] P. Ting, "An Efficient and Guaranteed Cold-Chain Logistics for Temperature-Sensitive Foods: Applications of RFID and Sensor Networks", International Journal of Information Engineering and Electronic Business, vol. 5 , no. 6, (2013).

[2] A. Rollo and M. Gnoni, "Business Performance Measurement and Management", Springer, Berlin Heidelberg (2010).

[3] G. Aiello, G. Scalia and R. MicaleI, "Simulation Analysis of Cold Chain Performance based on Time-temperature Data”, Production Planning and Control: The Manágement of Operations, vol. 23, no. 6 (2012).

[4] R. Bishara, "Cold Chain Management-An Essential Component of the Grobal Pharmaceutical Supply Chain", American Pharmaceutical Review, (2006) January/February.

[5] R. Heap, "Cold Chain Performance Issues now and in the Future", Bulletin of the IIR, vol. 4, (2006).

[6] D. Power and S. Kaparthi, "Building Web-based Decision-Support Systems", Studies in Informatics and Control, vol. 11, no. 4, (2002).

[7] H. Lee, "Creating Value through • Supply Chain Integration", http://www.highbeam.com/doc/1G1-64972562.html,(2000).

[8] R. Barton and A. Thomas, "Implementation of Intelligent Systems", Enabling Integration of SMEs to High-Value Supply Chain Networks. Engineering Applications of Artificial Intelligence, vol. 22, no. 6, (2009).

[9] D. Prajogo and J. Olhager, "Supply Chain Integration and Performance: The Effects of Long-term Relationships", Information Technology and Sharing, and Logistics Integration. International Journal of Production Economics, vol. 135, no. 1, (2012)

[10] S. Wamba and A. Chatfield, AContingency Model for Creating Value from RFID Supply Chain Network Projects in Logistics and Manúfacturing Environments", European Journal of Information Systems, vol. 18, no. 6, (2009).

[11] E. Abad, F. Palacio, M. Nuin, A Gonzalez De Zarate, A. Juarros, J. Gomez and S. Marco, "RFID Smart Tag for Traceability and Cold Chain Monitoring of Foods: Demonstration in an Intercontinental Fresh Fish Logistic Chain”, Journal of Food Engineering, vol. 93, no. 4, (2009).

[12] M. Aung, F. Liang, Y. Chang, C. Makatsoris and J. Chang, "RFID-and WSN-based Intelligent Cold Chain Management", International Journal of Manufacturing Research, vol. 6, no. 2, (2011).

[13] R. Montanari, "Cold Chain Tracking: A Managerial Perspective", Trends in Food Science and Technology, vol. 19, no. $8,(\mathbf{2 0 0 8})$

[14] J. Kuo and M. Chen, "Developing an Advanced Multi-Temperature Joint Distribution System for the Food Cold Chain”, Food Control, vol. 21, no. 4, (2010).

[15] D. Matthjas, M Robertson, M. Garrison and S. Newland, "Freezing Temperatures in the Vaccine Cold Chain: a Systematic Literature Review", Vaccine, vol. 25, no. 20, (2007).

[16] W Klibi, A. Martel and A. Guitouni, "The Design of Robust Value-Creating Supply Chain Networks: A Critical Review", European Journal of Operational Research, vol. 203, no. 2, (2010).

[17] T. Wirkas, S. Toikilik, N. Miller, C. Morgan and C. Clements, "A Vaccine Cold Chain Freezing Study in PNG Highlights Technology Needs for Hot Climate Countries", Vaccine, vol. 25, no. 4, (2007).

[18] N. Turner, A. Laws and L. Roberts, "Assessing the Effectiveness of Cold Chain Management for Childhood Vaccines", Journal of Primary Health Care, vol. 3, no. 4, (2011).

[19] R. Joshi, D. Banwet and R. Shankar, "A Delphi-AHP-TOPSIS based Benchmarking Framework for Performance Improvement of a Cold Chain”, Expert Systems with Applications, vol. 38, no. 8, (2011).

[20] G. Aiello, G. Scalla and R. Micale, "Simulation Analysis of Cold Chain Performance based on Time-temperature Data", Production Planning and Control: The Management of Operations, vol. 23, no. 6, (2012).

[21] H. Rediers, M. Claes, L. Peeters and K. Willems, "Evaluation of the Cold Chain of Fresh-cut Endive from Farmer to Plate", Postharvest Biology and Technology, vol. 51, no. 2, (2009).

[22] V. Raab, S. Bruckner, E. Beierle, Y. Kampmann, B. Petersen and J. Kreyenschmidt, "Generic Model for the Prediction of Remaining Shelf Life in Support of Cold Chain Management in Pork and Poultry Supply Chains", Journal on Chain and Network Science, vol. 8, no. 1, (2008). 
International Journal of Multimedia and Ubiquitous Engineering

Vol.9, No.8 (2014)

[23] S. James and C. James, "The Food Cold-Chain and Climate Change", Food Research International, vol. 43, no. 7, (2010).

[24] A. Vanooyen and B. Nienhuis, "Improving the Convergence of the Back-propagation Algorithm", Neural Networks, vol. 53, no. 3, (1992).

[25] H. Kitano, "Designing Neural Networks Using Genetic Algorithms with Graph Generation System", Complex Systems, vol. 4, (1990).

[26] S. Haykin, "Neural Networks and Learning Machines", Pearson Academic, London (2008).

[27] D. Gong and S. Liu, "Holographic-based Model for Logistics Resources Integration", Studies in Informatics and Control, vol. 22, no. 4, (2013).

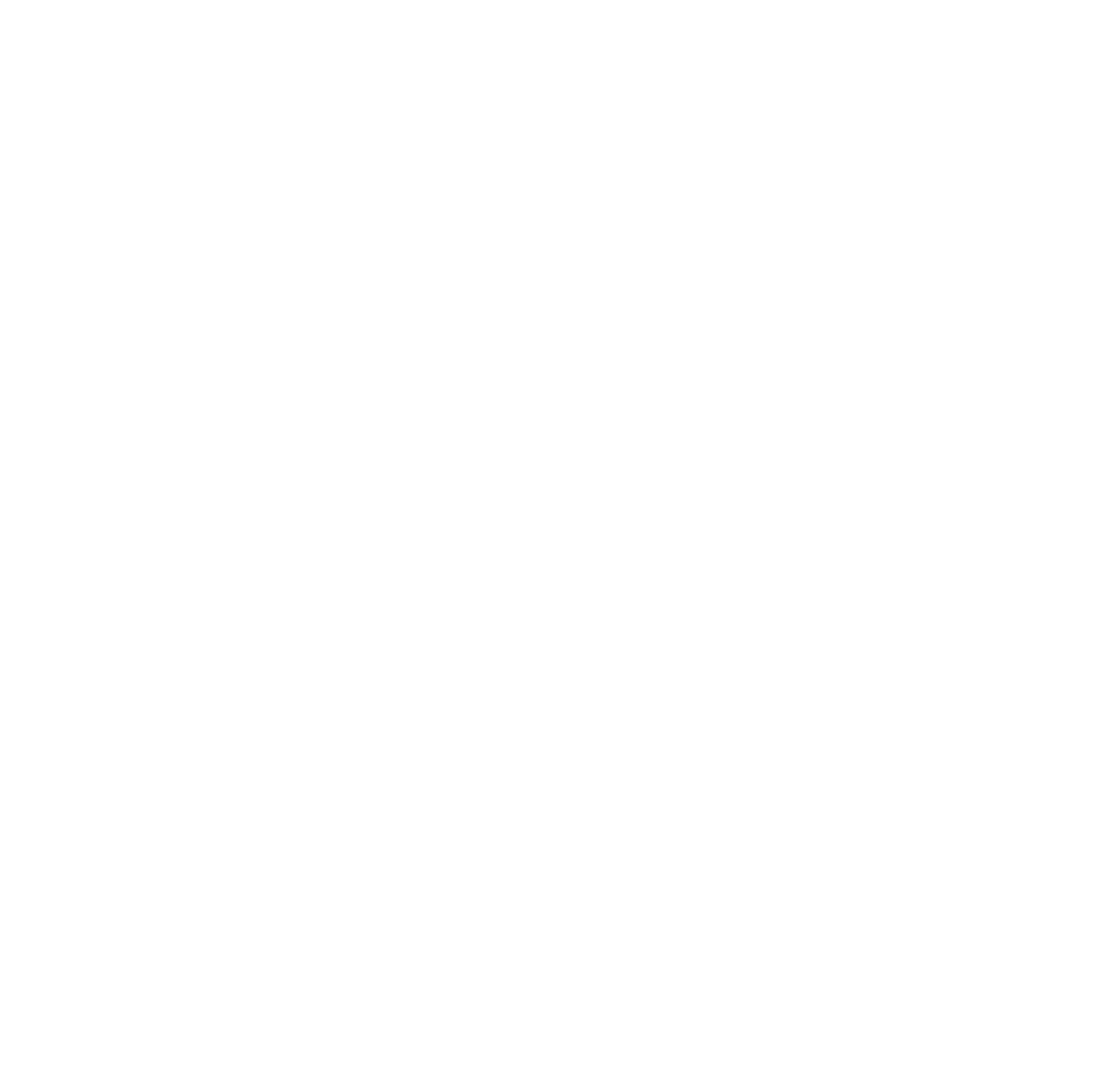

